\title{
Comparing Single and Hybrid methods of Deep Learning for Remaining Useful Life Prediction of Lithium-ion Batteries
}

\author{
Brahim Zraibi ${ }^{1}$, Mohamed Mansouri ${ }^{1}$, and Chafik Okar ${ }^{1,2}$ \\ ${ }^{1}$ Hassan First University of Settat, National School of Applied Sciences of Berrechid, Laboratory LAMSAD, Morocco \\ ${ }^{2}$ Carleton University, Ottawa, Canada
}

\begin{abstract}
The prediction lifetime of a Lithium-ion battery is able to be utilized as an early warning system to prevent the battery's failure that makes it very significant for assuring safety and reliability. This paper represents a benchmark study that compares its RUL prediction results of single and hybrid methods with similar articles. We suggest a hybrid method, named the CNN-LSTM, which is a combination of Convolutional Neural Network (CNN) and Long Short Term Memory (LSTM), for predicting and improving the accuracy of the remaining useful life (RUL) of Lithium-ion battery. We selected three statistical indicators (MAE, $\mathrm{R}^{2}$, and RMSE) to assess the results of performance prediction. Experimental validation is performed using the lithiumion battery dataset from the NASA and results reveal that the effectiveness of the suggested hybrid method in reducing the prediction error and in achieving better RUL prediction performance compared to the other algorithms.
\end{abstract}

Index Terms. Lithium-ion batteries, machine learning, remaining useful life, long short-term memory, convolutional neural network.

$\begin{array}{ll}\text { LIST OF ABBREVIATIONS } \\ \text { ANN } & \begin{array}{l}\text { artificial neural network } \\ \text { CNN }\end{array} \quad \begin{array}{l}\text { convolutional neural network } \\ \text { DNN }\end{array} \text { deep neural network } \\ \text { EOL } & \text { end of life } \\ \text { ESS } & \text { energy storage system } \\ \text { LSTM } & \text { long short-term memory } \\ \text { MAE } & \text { mean absolute error } \\ \text { ML } & \text { machine learning } \\ \text { NASA } & \text { national aeronautics and space administration } \\ \text { R } & \text { R-squared } \\ \text { RNN } & \text { recurrent neural network } \\ \text { RMSE } & \text { root mean square error } \\ \text { RUL } & \text { remaining useful life }\end{array}$

\section{Introduction}

Lithium-ion (Li-ion) batteries remain among the main sources of energy for EVs and electronic equipment and considered appropriate for the environment [1]. Thus, it considers a perfect choice for the energy storage system (ESS). In few years, they play major power sources in different areas e.g. consumer electronics, electric vehicles, aerospace electronics, and mobile communications [2]. Due to their best advantages: high-density of power and energy, suitable for the environment, low discharge rate, lightweight, speedy charge, and long lifetime [3][4]. However, there is the gradual deterioration of the battery during its lifetime, i.e. resistance increase and capacity decrease, for the reason that the external environment (e.g. discharge rates, temperatures), electrochemical reactions, and physical/chemical changes of the battery.

In spite of their advantages, degradation of battery performance over time can cause some losses such as catastrophic results of the devices, battery explosion of phones and EVs, rising maintenance costs, losses economic [5]. For avoiding these disasters, it requires more effort in lifespan prediction of $\mathrm{Li}$-ion batteries that are very necessary for improving the reliability and safety of the overall energy system however its future behavior prediction is a difficult task.

The battery management system (BMS) is necessary for ensuring the safety of Li-ion batteries, which is generally based on three essential elements: remaining useful life (RUL), state of charge (SOC), and state of health (SOH) which have a relationship respectively to the charge of the batteries and their aging [6]. The RUL is defined as the remaining number of cycles (charge/discharge) to get to the failure threshold, i.e. $70 \%$ of the nominal capacity, of the battery with a specific output capacity. Thus, for the RUL prediction of Li-ion battery, it can use four methods: direct measurement, model-based, data-driven, and hybrid methods [7]. 
The first method is the direct measurement, which uses to calculate the capacity and impedance of battery cells using the open-circuit voltage and Electrochemical Impedance Spectroscopy, respectively. The second method is model-based that uses different models such as equivalent circuit, electrochemical, or empirical models (Kalman filter, unscented Kalman filter, particle filter, etc.). The third method is data-driven prediction methods like artificial neural networks (ANN), support vector machines (SVM), and relevance vector machine (RVM), etc. [8], the advantage of this method is using the historical data that help for avoiding the necessity of complex physical or mathematical models for battery capacity degradation [9]. While the fourth method is the hybrid methods which combine the previous methods with each other [3] [10].

Several researchers have depended on hybrid neural network methods to predict the RUL of Li-ion batteries. Their performance results showed a high prediction accuracy compared to single methods. Wu et al. presented a method composed of the feedforward neural network (FFNN) and importance sampling (IS). Young et al. applied ELM in two approaches, which combine with MPSO, i.e. MPSO-ELM [9], and combine with HKA, i.e. HKA-ELM [10], for improving the stochastic parameters of the ELM to achieve a good prediction accuracy. While Chen et al. [11] presented a method called ELMBSASVM, which fuse ELM with a backtracking spiral algorithm (BSA) and support vector machines (SVM). Ren et al. [12] present a combined Autoencoder with Deep Neural Network (ADNN). Cadini et al. [13] presented a hybrid method that combines multi-layer perceptron and particle filter (MLP-PF). Fan et al. [3] proposed a mixedmethod named HA-FOSELM, which combines the Forgetting Online Sequential (FOS), Extreme Learning Machine (ELM), and the Hybrid Grey Wolf Optimizer (HGWO) algorithm. Wang et al. [14] used a method of the ensemble empirical mode decomposition EEMD with nonlinear autoregressive neural networks NARNN to predict RUL battery. Zhang et al. [15] propose a method fusing the partial incremental capacity and ANN. Cui et al. [16] present a hybrid method that combines unscented Kalman filter UKF, LSTM, and NN model. Yang et al. [17] proposed a mixed-method named CNN-BiLSTM, which combines CNN and bidirectional Long Short Term Memory (BiLSTM). Jia et al.[18] used a Wavelet neural network (WNN) with an unscented particle filter (UPF) to predict the RUL battery. Li et al. [20] and Ma et al. propose a hybrid neural network method, the first combines the Elman with LSTM [19], and the second combines CNN with LSTM [4].

The main contributions of this paper are to build a forecast model for RUL prediction of Li-ion batteries based on a hybridization technique by combining $\mathrm{CNN}$,
LSTM. To the best authors' knowledge, this is the first attempt to predict RUL Li-ion battery using CNN-LSTM based on univariate time series. Additionally, this work is to provide deeper insights on the single and hybrid methods for predicting the RUL of Li-ion battery by the comparison between methods of our work i.e., LSTM, CNN-LSTM, and methods in other papers. The proposed method obtained excellent results and achieved high predictive accuracy for the RUL estimation. Thus, it can help that to improve the lifetime control strategies and safety monitoring function of the battery for avoiding catastrophes.

The other sections of this paper are prepared as follows: Section II presents the architecture of CNN-LSTM. Section III introduces the RUL estimation techniques using the different methods. Section IV shows the experiment results and comparative study. Finally, a conclusion is given.

\section{Time series prediction using hybrid neural networks method}

\subsection{Related work of CNN-LSTM method}

Deep learning methods have already been used in many papers, which have known success in many areas especially in time-series prediction, where they gave a good performance due to their good advantages e.g. selfadaptive, and capability dependence of nonlinear.

Recently, the CNN-LSTM hybrid method has used in many studies because has realized good results when it applied in various fields. Among the fields that contributed is the medicine domain, ( e.g. forecast of haemorrhage into the cranium [20]), the plant domain, (e.g. estimating the characteristics of the plant [21] and recognizing the different headlines of Clickbait and classify it [22]), the pollution of air domain, (e.g. achieving a good prediction accuracy of air quality [23]), the financial domain, (e.g. estimating the volatility of gold [24]), the traffic domain, (e.g. avoiding the overcrowding of traffic [25]), the energy domain, (e.g. predicting the structure of energy at the following years [26], estimating the RUL and SOC of the batteries [27]), and more application domains. Depending on the good results shown by the CNN-LSTM hybrid method in previous works mentioned above. We propose to use it in our work to predict the RUL of Li-ion batteries for improving the performance of prediction accuracy of the same task.

\subsection{The CNN-LSTM method}

Every one of the CNN and LSTM has many advantages, thus, the combination of these networks in unite framework able to obtain good results of the RUL prediction of Li-ion batteries.

$\mathrm{CNN}$ is capable to capture the spatial relationship, extract local features and reduce the amount of the weights using the shared weights structure, and additional advantages such as the local dependency and scale invariance. Its structure includes the convolutional layer, 
which extracts different features of input through the convolution process, which contains several feature planes and neurons. The pooling layer extracts the secondary features, where it reduces the feature resolution and its surface dimension to obtain constant spatial features. The fully connected layers, and output layers. Both previous layers are congruous with each other since the pooling layer inputs are the outputs of the convolution layer. The fully connected layers are able to fuse the information from the previous layers. The final layer is the output layer that receives outputs of fully connected. The CNN is implemented using a kernel " $\mathrm{K}$ " or filter for obtaining a feature map $\mathrm{S}$ from the input vector $\mathrm{A}$. The formula is:

$$
S(\mathrm{i}, \mathrm{j})=\sum_{m} \sum_{n} A(i-m, i-n) \cdot K(m, n)
$$

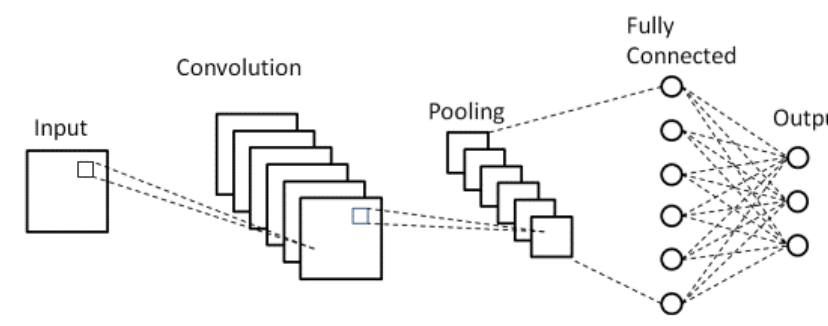

Fig.1. The architecture of the CNN

Recurrent Neural Networks (RNNs) are a category of ANN where they have an advantage different from DNNs that is the internal memory, which leads to allowing information to continue and to remember past information. It profits the temporal correlations between neurons and is utilized to treat the tasks that include the sequence of the features. Thus discovering the best way to make the next estimation reasonable. Nevertheless, it has a problem with the long-distance dependencies that lead to disappear gradients and vanishing. To avoid these problems, LSTM is used to control the propagation of gradients information and remembering the parameters as input during the long term and it also has the addition operation that leads to solving the problem of disappearing gradient. The LSTM architecture is containing three gates i.e. the input (i), forget (f), and output (o), as well as a memory unit.

The LSTM is the cell of the LSTM consists of a longterm state of $\mathrm{Ct}$ and a short-term state ht. The calculation of the hidden layer nodes depends on the input of the current layer and the activation values of nodes at the previous moment. LSTM's equations can be defined as follows:

$$
\begin{gathered}
f_{t}=\sigma\left(W_{f}\left[h_{t-1}, x_{t}\right]+b_{f}\right) \\
\mathrm{i}_{\mathrm{t}}=\sigma\left(W_{i}\left[h_{t-1}, x_{t}\right]+b_{i}\right) \\
q_{t}=\tanh \left(W_{q}\left[h_{t-1}, x_{t}\right]+b_{q}\right) \\
o_{t}=\sigma\left(W_{o}\left[h_{t-1}, x_{t}\right]+b_{o}\right. \\
c_{t}=f_{t} * c_{t-1}+i_{t} * q_{t} \\
h_{t}=o_{t} * \tanh \left(c_{t}\right)
\end{gathered}
$$

$\mathrm{W}$ is the weight matrices, $\mathrm{b}$ is the bias, $\sigma$ is the sigmoid function, $\mathrm{x}_{\mathrm{t}}$ is the unit input at time $\mathrm{t}, \mathrm{h}_{\mathrm{t}-1}$ is the unit output of the previous LSTM cell, $c_{t}$ and $c_{t-1}$ are the cell states at time $\mathrm{t}$ and $\mathrm{t}-1$, respectively, $\mathrm{q}_{\mathrm{t}}$ is the hyperbolic function.

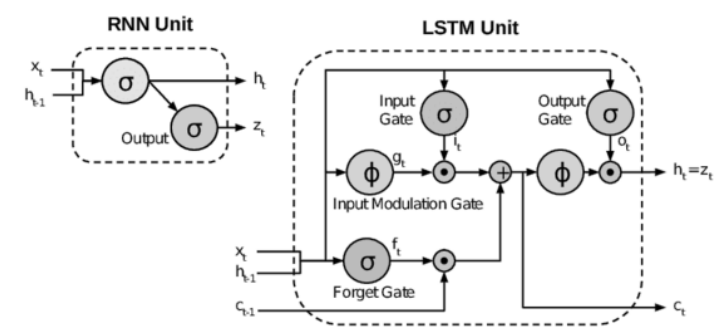

Fig.2. The architecture of each the RNN and LSTM.

The combination CNN-LSTM algorithm profit the advantages of CNN and LSTM. It able to extract two types of features are the spatial and temporal. The first is the interrelations within current inputs, while the second is the correlations between current RUL and past inputs. Thus, the proposed method is designed to take advantage of all of them; this architecture of the proposed method shown in fig. 3 is designed to profit from all previous advantages mentioned above.

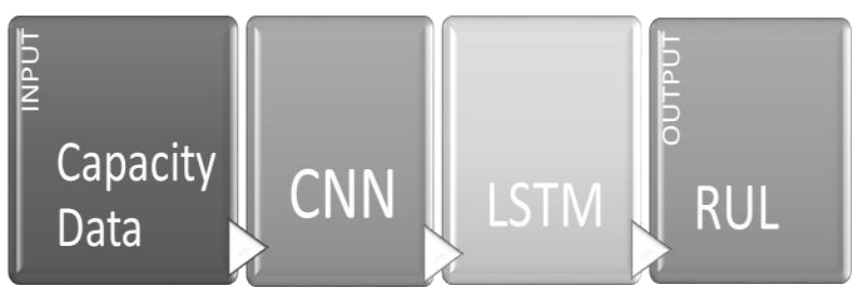

Fig.3. The framework of the proposed method.

\section{RUL PREDICTION}

In this section, we will present the results of ours experimentations to predict RUL with three algorithms: RNN, LSTM, and CNN-LSTM. The experimental data of the NASA Prognostics Center of Excellence [31] is used in this paper for validation. It consists of aging data for 18650 Li-ion batteries of $2 \mathrm{Ah}$ rated capacity. Table.1 introduce the information about this battery as follow:

Table 1. The description of NASA Li-ion battery

\begin{tabular}{|c|c|}
\hline battery & B0006 \\
\hline Type & $18650 \mathrm{NMC}$ \\
\hline Constant charge current & $1.5 \mathrm{~A}$ \\
\hline Minimal charge current & $20 \mathrm{~mA}$ \\
\hline Discharge current & $2 \mathrm{~A}$ \\
\hline nominal capacity & $2 \mathrm{Ah}$ \\
\hline Charge/Discharge cut-off voltage & $4.2 / 2.5 \mathrm{~V}$ \\
\hline charge/discharge cycles & 168 \\
\hline
\end{tabular}

Table 2. Hardware and software environment

\begin{tabular}{|c|c|}
\hline $\begin{array}{c}\text { Hardware and Software } \\
\text { Environment }\end{array}$ & Version or Model Number \\
\hline Operating System & Windows 10 professional edition \\
\hline Development Environment & Python with Tensorflow \\
\hline CPU & i7-8565U \\
\hline RAM & $8 \mathrm{G}$ \\
\hline Processor & $1.80 \mathrm{GHz}$ CPU \\
\hline GPU & Intel(R) HD Graphics Family \\
\hline
\end{tabular}


The B0006 battery dataset, shown in Figure 4, contains 168 cycles, 80 of them are utilized for training and the rest for validation. The hardware and software environment shown in Table 2 was used to implement the three methods, i.e. RNN, LSTM, and CNN-LSTM, The rectified linear unit (ReLU) activation function is used along with Adam optimizer. Huber loss is also employed. Besides, to evaluate the RUL prediction performance of the algorithms, we use the mean absolute error (MAE) [28], root mean square error (RMSE) and $R$ square $\left(R^{2}\right)$ [29]. They are defined in equations 3,4 and 5 .

$$
\begin{aligned}
& M A E=\frac{1}{K} \sum_{k=1}^{k}\left|y_{k}-\widehat{y_{k}}\right| \\
& R M S E=\sqrt{\frac{1}{N} \sum_{i=1}^{N}\left(y_{k}-\widehat{y_{k}}\right)^{2}} \\
& R^{2}=1-\frac{\sum_{K=1}^{n}\left(y_{k}-\widehat{y_{k}}\right)^{2}}{\sum_{K=1}^{n}\left(y_{k}-\widehat{y_{k}}\right)^{2}}
\end{aligned}
$$

Where $y_{k}$ is the true value battery capacity, while $\widehat{y_{k}}$ is the estimated value one, $\overline{y_{k}}$ represents the average of actual one. When the MAE and RMSE it is close to zero, the capacity prediction accuracy is higher. As for $\mathrm{R}^{2}$, a value close to one yields better accurate RUL prediction results

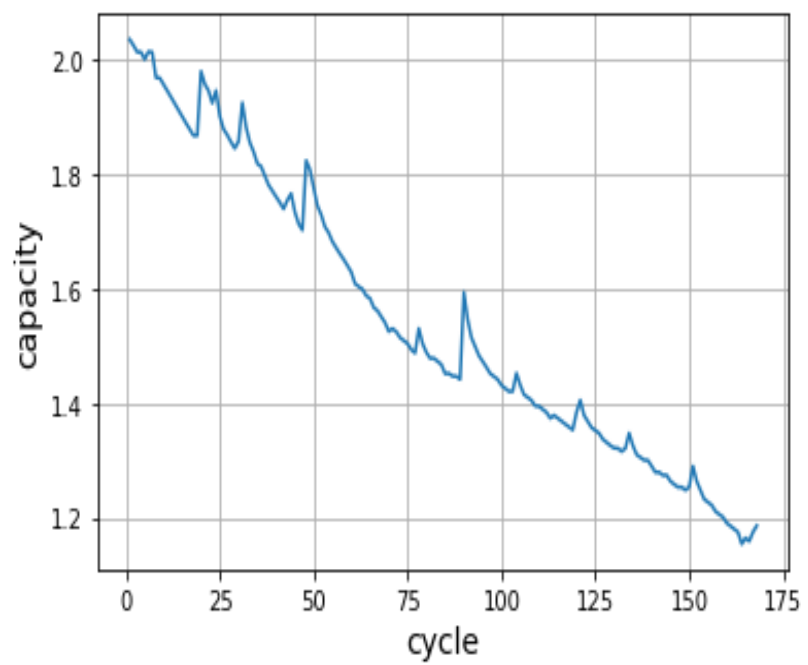

Fig. 4. Capacity degradation curve of battery B0006

Each algorithm consists of four steps: data preprocessing, algorithm training (80 cycles), validation estimation ( 88 cycles) and 40 new predictions cycles (from 169 to 208).

Below, the RUL prediction results are presented for two algorithms the real value is represented in blue color, forecast validation in green color that is starting in the 80 cycle, and new forecast results of these algorithms in red color.

\subsection{RUL estimation with the RNN method}

First algorithm is RNN, which is used to predict the RUL of Li-ion battery. Fig. 5 reveals RUL estimation performance for the B0006 Li-ion battery. It can be observed that the curve of validation is near the curve of true value, which makes this algorithm good learned. Nevertheless, it has also a bad new prediction. The MAE decreases gradually with the number of epochs. The MAE and RMSE values of RUL estimation are equal to 0.01594 and 0.02903; In addition, the $\mathrm{R}^{2}$ value is equal to 0.917 .

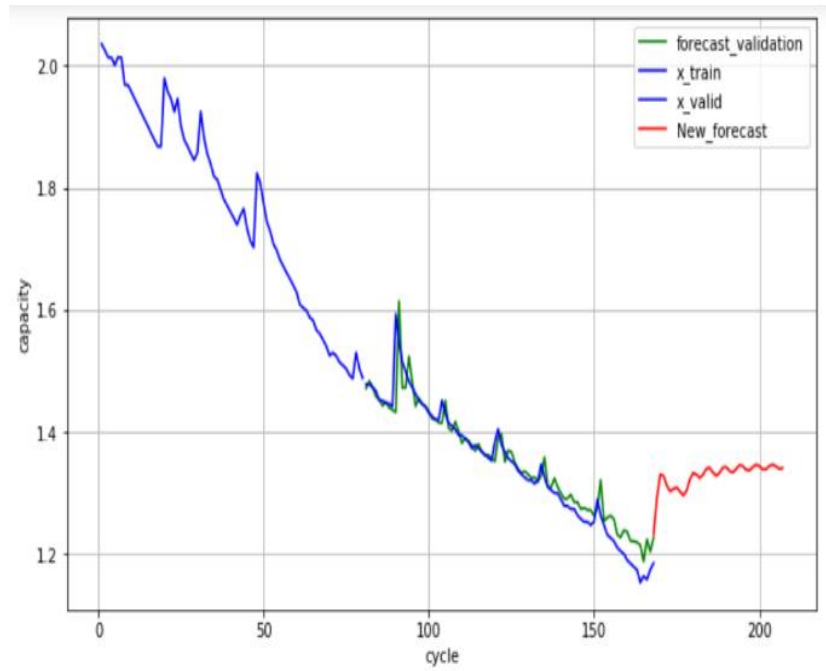

Fig. 5. (a) RUL prediction results using RNN
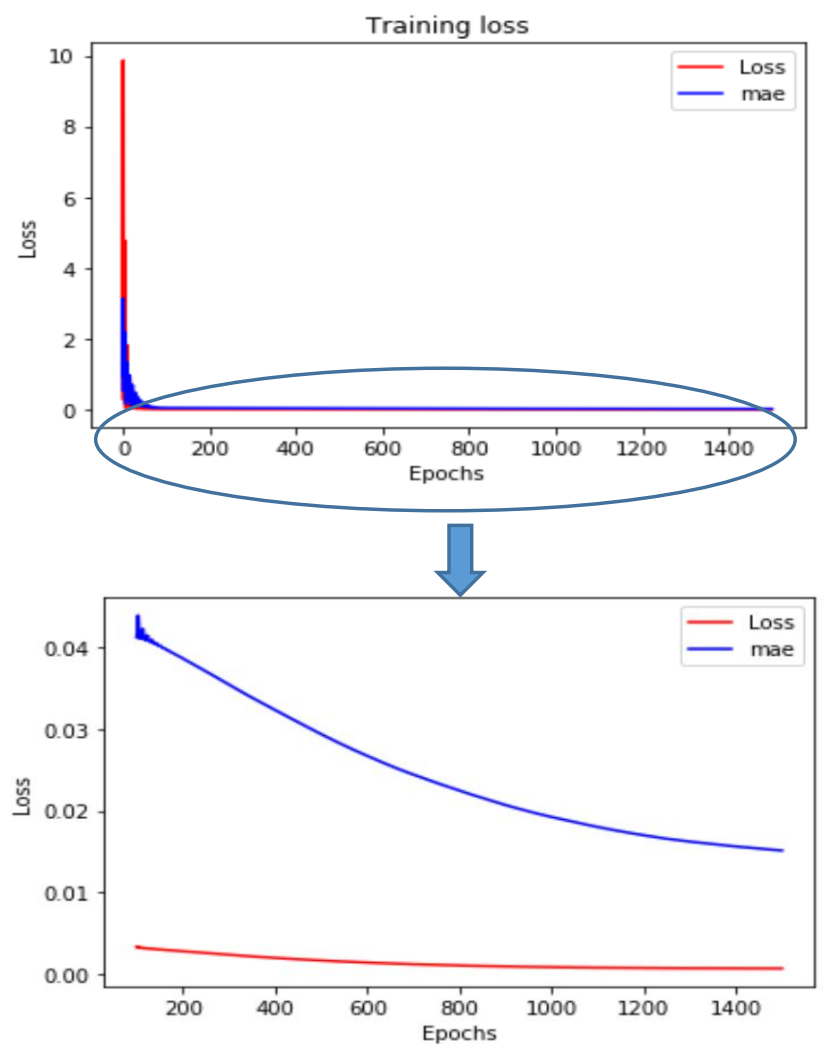

Fig. 5. (b) RUL training performance using RNN

\subsection{RUL estimation with the LSTM method}

A second algorithm, namely LSTM, is used for the same task. Fig. 6 reveals RUL estimation performance. A better accuracy is obtained in the validation phase. The MAE 
decreases gradually with the number of epochs. The MAE and RMSE values of RUL estimation are equal 0.01299 and 0.02240, respectively, which is lower than those obtained with the RNN. While, the $\mathrm{R}^{2}$ value is 0.950 that is high with respect to the RNN. However, it overcome to $\mathrm{RNN}$, the prediction performance of new forecasts is still not satisfactory.

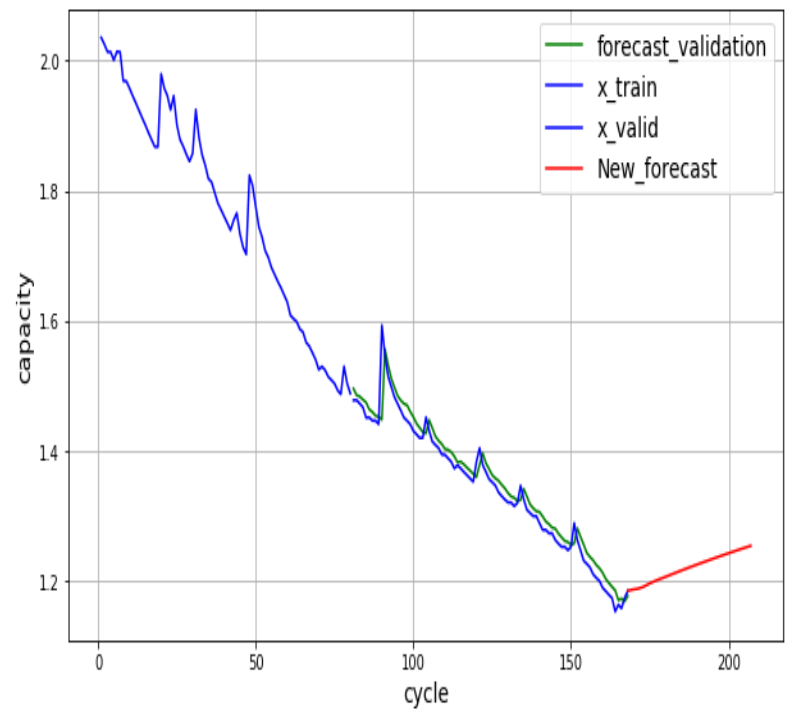

Fig. 6. (a) RUL prediction results using LSTM
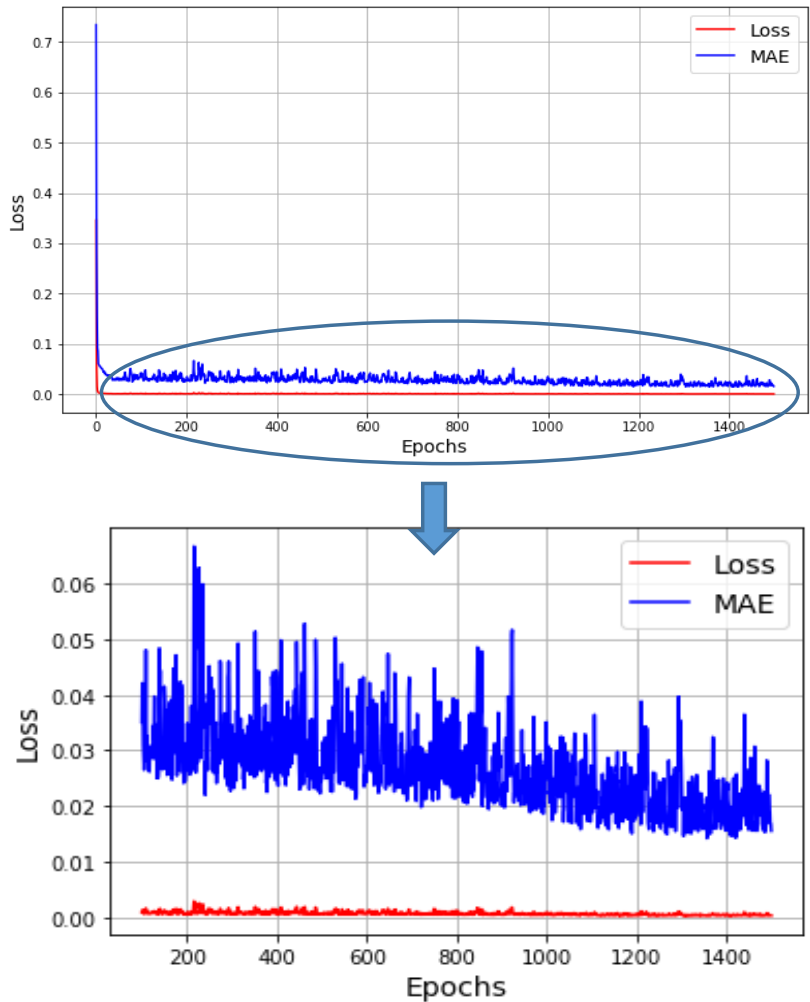

Fig. 6. (b) RUL training performance using LSTM

Next, hybrid algorithm combining the CNN, and LSTM, which are proposed to improve further the estimation performance and more importantly to achieve acceptable prediction for new forecasts.

\subsection{RUL estimation with the CNN-LSTM method}

The CNN-LSTM hybrid algorithm is introduced as a combination between CNN and LSTM for the RUL prediction of Li-ion battery.

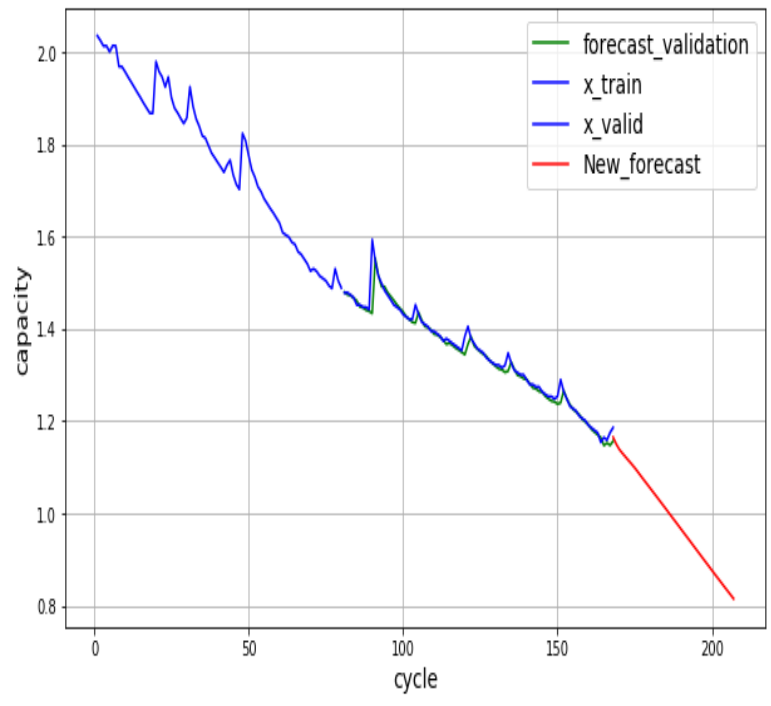

Fig. 7. (a) RUL prediction results using CNN-LSTM
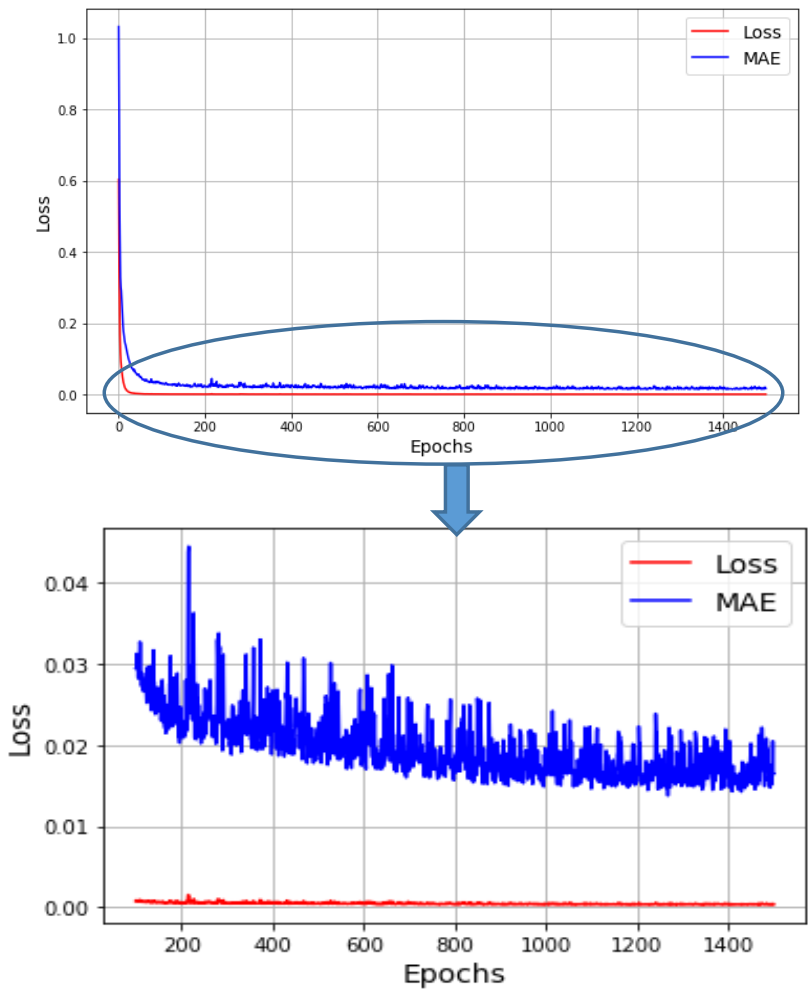

Fig.7. (b) RUL training performance using CNN-LSTM.

It is clear from Fig. 7 that there is better consistency between the estimates and the true values. Moreover, a good performance is finally obtained for the prediction of new forecasts. In addition, the MAE curve does converge to the loss curve that remains close to zero in spite the high fluctuations. The MAE, $\mathrm{R}^{2}$, and RMSE values are equal 
$0.0090,0.957$ and 0.02092 , respectively, which are the lowest values obtained compared to previous methods.

This experiment demonstrates clearly the superiority of hybrid method over single methods in achieving high estimation accuracy and more importantly in predicting new forecasts. Thus, CNN-LSTM achieve the highest accuracy in the RUL prediction of the Li-ion battery. Next section summarizes the numeric RUL prediction errors and put them in perspective with other the best performant methods in literature.

\section{Comparative Results Analysis}

\subsection{RUL validation and the evaluation criteria}

The above experiments reveal that hybrid method has successfully learned the dynamic nature of Li-ion batteries. Table 3 summarizes the three indicators used to evaluate prediction performance, which are the MAE, the $\mathrm{R}^{2}$ and the RMSE.

Table 3. RUL estimation results for B0006

\begin{tabular}{|l|l|l|l|}
\hline Methods & MAE & $\mathbf{R}^{2}$ & RMSE \\
\hline RNN & 0.01594 & 0.917 & 0.02903 \\
\hline LSTM & 0.01299 & 0.950 & 0.02240 \\
\hline CNN-LSTM & 0.00902 & 0.957 & 0.02092 \\
\hline
\end{tabular}

Table 3 shows the performance of the proposed algorithms with the same starting point for testing sets for battery B0006, where the MAE and RMSE values with hybrid method are lower than that with single ones and the $\mathrm{R}^{2}$ values with hybrid method are higher than that of single ones. This confirms that proposed hybrid method improve substantially the RUL estimation. In addition, the improvement from CNN-LSTM to LSTM is $30.5 \%$ of MAE and $6.6 \%$ of RMSE.

\subsection{Results analysis and comparison}

This section presents a comparative analysis of the RUL estimation accuracy between various comparable methods in literature among the single and hybrid methods. The single seem to be not adequate for time series data compared to the hybrid. This is revealed by the findings summarized in Table 3 and in the comparative analysis presented in this section.

In order to compare more generally with other types of neural networks of prediction methods, we extract some results of performance methods from other papers, where their methods have the same dataset, i.e. NASA, and the same indicators of performance.
Table 4. RUL estimation results of B0006 for some papers

\begin{tabular}{|l|l|l|l|}
\hline Methods & MAE & $\mathbf{R}^{\mathbf{2}}$ & RMSE \\
\hline RNN & 0.01594 & 0.917 & 0.02903 \\
LSTM & 0.01299 & 0.950 & 0.02240 \\
CNN-LSTM & 0.00902 & 0.957 & 0.02092 \\
\hline SC-CNN & 0.0623 & & 0.0701 \\
SC-LSTM [30] & 0.0210 & & 0.0288 \\
\hline RNN & & & 0.1131 \\
LSTM & & & 0.0784 \\
HA-FOSELM [3] & & & 0.0434 \\
\hline ELM & & 0.8829 & \\
PSO-ELM & & 0.8945 & \\
MPSO-ELM [9] & & 0.9514 & \\
\hline UKF & 0.0994 & & 0.1275 \\
AUKF & 0.0371 & & 0.0489 \\
AUKF-GASVR [29] & 0.0368 & & 0.0483 \\
\hline
\end{tabular}

Table 4 reveals that the accuracy of all hybrid methods exceeds the one obtained with their single counterparts. This shows the power of hybridization in achieving better results and confirms the findings presented in this manuscript. In fact, hybridization is observed to decrease MAE, RMSE, and $\mathrm{R}^{2}$.

According to the above analysis, we can deduce that the proposed CNN-LSTM RUL prediction approach is an excellent estimator with its high accuracy.

\section{Conclusion}

In this paper, a hybrid CNN-LSTM algorithm is suggested by combining two well-known algorithms, i.e., Convolutional Neural Networks (CNNs) and Long Short Term Memory (LSTM) to predict the remaining useful life (RUL) and improve the prediction of the Li-ion batteries. The proposed method is experimentally validated on a dataset obtained from NASA. Experimental results demonstrate the high RUL prediction capability of the Liion battery. Moreover, the prognostic of the proposed hybrid method is more accurate than single ones. Generally, three prediction performance indices reveal the highest accuracy of CNN-LSTM compared to RNN, LSTM, and other existing methods.

\section{References}

1. Chaoui H, Ibe-Ekeocha CC (2017) State of Charge and State of Health Estimation for Lithium Batteries Using Recurrent Neural Networks. IEEE Trans Veh Technol 66:8773-8783. https://doi.org/10.1109/TVT.2017.2715333

2. Luo W, Lv C, Wang L, Liu C (2011) Study on impedance model of Li-ion battery. Proc 2011 6th IEEE Conf Ind Electron Appl ICIEA 2011 19431947.

https://doi.org/10.1109/ICIEA.2011.5975910

3. Fan J, Fan J, Liu F, et al (2019) A Novel Machine Learning Method Based Approach for Li-Ion Battery Prognostic and Health Management. IEEE 
Access

7:160043-160061

https://doi.org/10.1109/ACCESS.2019.2947843

4. Ma G, Zhang Y, Cheng C, et al (2019) Remaining useful life prediction of lithium-ion batteries based on false nearest neighbors and a hybrid neural network. Appl Energy 253:113626. https://doi.org/10.1016/j.apenergy.2019.113626

5. Yu P, Hou Y, Song Y, et al (2018) Lithium-Ion Battery Prognostics with Hybrid Gaussian Process Function Regression. Energies 11: https://doi.org/10.3390/en11061420

6. Hu X, Jiang J, Cao D, Egardt B (2016) Battery health prognosis for electric vehicles using sample entropy and sparse Bayesian predictive modeling. IEEE Trans Ind Electron 63:2645-2656. https://doi.org/10.1109/TIE.2015.2461523

7. Zhou J, Liu D, Peng Y, Peng X (2012) Dynamic battery remaining useful life estimation: An online data-driven approach. 2012 IEEE I2MTC - Int Instrum Meas Technol Conf Proc 2196-2199. https://doi.org/10.1109/I2MTC.2012.6229280

8. Xiong R (2020) Battery Management Algorithm for Electric Vehicles

9. Liu D, Luo Y, Liu J, et al (2014) Lithium-ion battery remaining useful life estimation based on fusion nonlinear degradation AR model and RPF algorithm. Neural Comput Appl 25:557-572. https://doi.org/10.1007/s00521-013-1520-x

10. Duan B, Zhang Q, Geng F, Zhang C (2020) Remaining useful life prediction of lithium-ion battery based on extended Kalman particle filter. Int J Energy Res 44:1724-1734. https://doi.org/10.1002/er.5002

11. Chen X-D, Yang H-Y, Wun J-S, et al (2020) Life prediction of lithium-ion battery based on a hybrid model. Energy Explor Exploit 014459872091172. https://doi.org/10.1177/0144598720911724

12. Ren L, Zhao L, Hong S, et al (2018) Remaining Useful Life Prediction for Lithium-Ion Battery: A Deep Learning Approach. IEEE Access 6:5058750598.

https://doi.org/10.1109/ACCESS.2018.2858856

13. Cadini F, Sbarufatti C, Cancelliere F, Giglio M (2019) State-of-life prognosis and diagnosis of lithium-ion batteries by data-driven particle filters. Appl Energy 235:661-672. https://doi.org/10.1016/j.apenergy.2018.10.095

14. Wang P, Dan X, Yang Y (2019) A multi-scale fusion prediction method for lithium-ion battery capacity based on ensemble empirical mode decomposition and nonlinear autoregressive neural networks. Int $\mathrm{J}$ Distrib Sens Networks 15:. https://doi.org/10.1177/1550147719839637

15. Zhang S, Zhai B, Guo X, et al (2019) Synchronous estimation of state of health and remaining useful lifetime for lithium-ion battery using the incremental capacity and artificial neural networks. J Energy Storage 26:100951. https://doi.org/10.1016/j.est.2019.100951

16. Cui X, Hu T (2020) State of Health Diagnosis and Remaining Useful Life Prediction for Lithium-ion Battery Based on Data Model Fusion Method.
IEEE Access 8:207298-207307. https://doi.org/10.1109/ACCESS.2020.3038182

17. Yang H, Wang P, An Y, et al (2020) Remaining useful life prediction based on denoising technique and deep neural network for lithium-ion capacitors. eTransportation 5:100078. https://doi.org/10.1016/j.etran.2020.100078

18. Jianfang J, Keke W, Xiaoqiong P, et al (2021) Multi-Scale Prediction of RUL and $\mathrm{SOH}$ for Lithium-Ion Batteries Based on WNN-UPF Combined Model. Chinese J Electron 30:26-35. https://doi.org/10.1049/cje.2020.10.012

19. Li X, Zhang L, Wang Z, Dong P (2019) Remaining useful life prediction for lithium-ion batteries based on a hybrid model combining the long shortterm memory and Elman neural networks. J Energy Storage 21:510-518. https://doi.org/10.1016/j.est.2018.12.011

20. Nguyen TN, Tran QD, Nguyen TN, Nguyen QH (2020) A CNN-LSTM Architecture for Detection of Intracranial Hemorrhage on CT Scans. medRxiv 2020.04.17.20070193.

https://doi.org/10.1101/2020.04.17.20070193

21. Yasrab R, Pound MP (2020) PhenomNet: Bridging phenotype-genotype gap: A CNN-LSTM based automatic plant root anatomization system. bioRxiv 2020.05.03.075184.

https://doi.org/10.1101/2020.05.03.075184

22. Kaur S, Kumar P, Kumaraguru P (2020) Detecting clickbaits using two-phase hybrid CNN-LSTM biterm model. Expert Syst Appl 151:113350. https://doi.org/10.1016/j.eswa.2020.113350

23. Zhang Q, Lam JC, Li VO, Han Y (2020) DeepAIR: A Hybrid CNN-LSTM Framework forFineGrained Air Pollution Forecast. 1-7

24. Vidal A, Kristjanpoller W (2020) Gold volatility prediction using a CNN-LSTM approach. Expert Syst Appl 157:. https://doi.org/10.1016/j.eswa.2020.113481

25. Bogaerts T, Masegosa AD, Angarita-Zapata JS, et al (2020) A graph CNN-LSTM neural network for short and long-term traffic forecasting based on trajectory data. Transp Res Part C Emerg Technol 112:62-77.

https://doi.org/10.1016/j.trc.2020.01.010

26. Li Y, He Y, Zhang M (2020) Prediction of Chinese energy structure based on Convolutional Neural Network-Long Short-Term Memory (CNNLSTM). Energy Sci Eng 1-10. https://doi.org/10.1002/ese3.698

27. Song X, Yang F, Wang D, Tsui KL (2019) Combined CNN-LSTM Network for State-ofCharge Estimation of Lithium-Ion Batteries. IEEE Access 7:88894-88902. https://doi.org/10.1109/ACCESS.2019.2926517

28. Liu D, Luo Y, Peng Y, et al (2012) Lithium-ion battery remaining useful life estimation based on nonlinear AR model combined with degradation feature. Proc Annu Conf Progn Heal Manag Soc 2012, PHM 2012 336-342

29. Xue Z, Zhang Y, Cheng C, Ma G (2020) Remaining useful life prediction of lithium-ion 
batteries with adaptive unscented kalman filter and optimized support vector regression. Neurocomputing 376:95-102. https://doi.org/10.1016/j.neucom.2019.09.074

30. Choi Y, Ryu S, Park K, Kim H (2019) Machine Learning-Based Lithium-Ion Battery Capacity Estimation Exploiting Multi-Channel Charging Profiles. IEEE Access 7:75143-75152. https://doi.org/10.1109/ACCESS.2019.2920932

31. B. Saha and K. Goebel, "Battery data set", NASA AMES Prognostics Data Repository, USA, 2007. 